\title{
Structural Changes and Detection of Liver Fibrosis- Related Protein Levels in Coculture of Alveolar Echinococcosis-Protoscoleces and Human Hepatic Stellate Cells
}

DEping cao ( $\sim$ qhmccdp@163.com )

Guilin Medical college https://orcid.org/0000-0002-1973-8879

Emad Shamsan

Taiz University

Bofan Jiang

The Third hospital of Guangzhou medical univerisity

Zhang Yaogang

The Affiliated Hospital of Qinghai University

Mustafa Abdo Saif Dehwah

Guangzhou Medical University

\section{Research Article}

Keywords: Echinococcus multilocularis, hepatic stellate cell, liver fibrosis, protoscoleces, collagen- $\rrbracket, a-$ smooth muscle actin, osteopontin

Posted Date: March 22nd, 2021

DOl: https://doi.org/10.21203/rs.3.rs-324820/v1

License: (c) (i) This work is licensed under a Creative Commons Attribution 4.0 International License.

Read Full License 


\section{Abstract}

\section{Background}

Echinococcus multilocularis is a causative agent of human alveolar echinococcosis (AE). AE leads to cirrhosis in several organs, such as the liver, triggering severe conditions, including hepatic failure and encephalopathy. The main purpose of this study is to explore the interaction between treated hepatic stellate cells and AE-protoscoleces (AE-PSCs). The results of this study will be provided experimental basis for revealing the mechanisms of hepatic fibrosis after $A E$ infection.

Methods

We investigated the role of alveolar echinococcosis-protoscoleces (AE-PSCs) in liver fibrosis and structural changes and liver fibrosis-related protein expression in a coculture of PSCs and human hepatic stellate cells (HSCs). Structural changes were detected by transmission electron microscopy, whereas liver fibrosis-related proteins, collagen I, alpha-smooth muscle actin, and osteopontin levels were measured by western blotting and enzyme-linked immunosorbent assay.

Results

PSCs exhibited morphological changes, specifically changes in shape, and showed slight changes in the cytoplasmic membrane, whereas structural modifications were observed in HSCs. Additionally, western blotting and enzyme-linked immunosorbent assay revealed that PSCs treated in vitro with HSC-LX2

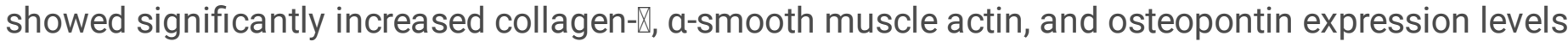
after 3-4 days of incubation in a coculture system. AE-PSCs induced liver fibrosis by inducing extracellular matrix expression and HSC activation.

\section{Conclusions}

These results provide insight into the pathogenesis of echinococcosis-induced hepatic fibrosis and introduce therapeutic targets and diagnostic criteria for managing echinococcosis-dependent liver fibrosis.

\section{Background}

Alveolar echinococcosis $(\mathrm{AE})$ is one of the deadliest human infections caused by Echinococcus multilocularis (E.m) [1-2] and is prevalent in most of the northern hemisphere [3-4]. Epidemiological studies have shown that $A E$ is common in central Asia including areas of Kyrgyzstan, Kazakhstan, and Western China [5-6]. Its adult worms are harbored in the small intestine of definitive hosts (such as wolves, foxes, dogs, and cats). The eggs are released in the feces of definitive hosts. Humans become infected by eating and drinking egg-contaminated food and water. Oncospheres in the eggs are incubated in the intestines of humans, after which they migrate to the liver and live as parasites. Next, they develop into microvesicles, producing large amounts of protoscolex (PSC) in the liver [7]. These PSCs invade the 
liver and trigger hepatic fibrosis. Hepatic cells at this phase are damaged by the toxic products of PSCs and their infiltration, making surgical resection difficult, and secondary infections typically occurred [8]. Patients with $A E$ can remain asymptomatic for up to 15 years [9]. AE is often delayed when a patient with $A E$ reports to the hospital. The parasite infection is fatal in $94 \%$ of infected people, particularly in untreated and undiagnosed patients [10-11]. Surgery and chemotherapy significantly improve the diagnosis of $A E$ but have limited effects. It is impossible to completely remove the tumor because of its growth. Therefore, understanding the mechanism underlying the interactions between parasites and humans and the pathogenesis of this disease is necessary to develop treatments for echinococcosisinduced liver damage.

Hepatic stellate cells (HSCs), which are the major cells responsible for forming extracellular matrix (ECM) proteins during liver cirrhosis, are found in the Disse space area and act as a major storage site for vitamin A. They are stimulated in response to growth factors, inflammatory stimuli, and, in the case of liver damage, oxidative stress. For example, damaged liver cells, resident phagocytic cells, infiltrating inflammatory cells, aggregated platelets, and Kupffer cells can activate HSCs. Activated HSCs differentiate into muscle fibroblasts, which express a-smooth muscle actin (SMA) to accelerate the cirrhosis process. Under pathological conditions of cirrhosis, HSCs lose their retinoid and synthesize a large mass of ECM components, including collagen, proteoglycans, and glycoproteins [12]. In addition, they undergo proliferation, migration, and increased ECM generation, including collagen-forming fibers, fibronectin, and proteoglycans, which lead to septa formation in the chronically damaged liver [13]. Moreover, an imbalance in the collagen fiber formation process can cause fibrosis [14]. Hepatic parasitic fibrosis caused by AE-PSCs is a host response associated with immune cell infiltration that activates HSC differentiation into fibroblasts [15]. Therefore, identifying the mechanisms underlying AE-induced liver cirrhosis may help reveal the disease pathogenesis and lead to the development of improved treatments.

In this study, AE-PSCs were cultured in modified media to investigate their role in liver cirrhosis. The human HSC-LX2 cell strain was cultured with AE-PSCs, and then liver cirrhosis-related proteins, such as Col-I, a-SMA, and osteopontin (OPN), were identified.

\section{Methods}

\section{Animal infection and parasite}

Mongolian gerbrils were infected with AE-PSCs for 7 months. The infected gerbrils with heavy infection was anesthetized with carbon dioxide. Masses of PSCs were collected from the killed jirds and washed with aseptic phosphate-buffered saline (PBS) under sterile conditions. After separating the jird tissue, the lesion tissue was mashed into small pieces using an aseptic sieve (mesh sieve, $300 \mu \mathrm{m}$ ) and passed through the sieve. AE-PSCs were washed five times with PBS containing $100 \mu \mathrm{g} / \mathrm{mL}$ streptomycin and $100 \mathrm{U} / \mathrm{mL}$ penicillin. The PSCs were then placed in sediments at room temperature $\left(25-30^{\circ} \mathrm{C}\right)$ for $5 \mathrm{~min}$. Next, the tissues were passed through a sieve (mesh sieve, $100 \mu \mathrm{m}$ ) and washed five times with PBS containing $100 \mu \mathrm{g} / \mathrm{mL}$ streptomycin and $100 \mathrm{U} / \mathrm{mL}$ penicillin at RT to remove debris. The viability of the 
PSCs was tested by staining with $0.1 \%$ trypan blue, and dead PSCs were stained as blue. Only PSCs showing $>90 \%$ viability were selected for further use [16]. PSCs were cultured in Dulbecco's modified Eagle's medium (DMEM) containing 10\% (v/v) fetal bovine serum (FBS) (Gibco, Grand Island, NY, USA), $0.45 \%(\mathrm{w} / \mathrm{v})$ yeast extract, $0.4 \%(\mathrm{w} / \mathrm{v})$ glucose, $1000 \mu \mathrm{g} / \mathrm{mL}$ streptomycin, and $1000 \mathrm{U} / \mathrm{mL}$ penicillin at $37^{\circ} \mathrm{C}$ in the presence of $5 \% \mathrm{CO}_{2}$.

\section{Cell culture}

Human HSCs were obtained from the Beijing University of Biological Sciences (Beijing, China). HSCs were preserved in DMEM containing Nutrient Mixture F-12 (Gibco) supplemented with 10\% FBS. The cell culture was maintained and cultured in DMEM supplemented with $10 \%$ FBS containing $1000 \mathrm{U} / \mathrm{mL}$ penicillin $\mathrm{G}$ and $1000 \mu \mathrm{g} / \mathrm{mL}$. When the cultures reached confluence, they were trypsinized and passaged at a ratio of 1:3.

\section{Co-culture of HSC-LX2 and protoscoleces PSCs}

HSCs were plated into 40 Petri dishes $\left(60 \mathrm{~mm}, 1.5 \times 10^{5}\right.$ cells/plate) and divided into five groups. Three groups were treated with PSCs and the other two groups were used as controls (HSCs only) and PSCs only. The three groups of HSCs were treated with PSCs and cultured at ratios of 1:200 (1 PSC: 200 HSCs), 2:200 (2 PSCs: $200 \mathrm{HSCs}$ ), and 3:200 (3 PSCs: $200 \mathrm{HSCs}$ ). All groups were incubated at $37^{\circ} \mathrm{C}$ in the presence of $5 \% \mathrm{CO}_{2}$ for 24,48 , and $72 \mathrm{~h}$. The harvested HSC-LX2 cells were divided into two groups. Samples in the first group were centrifuged, and the pellet was collected to measure the expression of Col-I, a-SMA, and OPN. The co-culture supernatant was collected for enzyme-linked immunosorbent assay (ELISA), and that of the other group was collected to analysis of structural changes.

\section{Transmission electronic microscopy}

Cultured human HSCs and PSCs were examined by transmission electron microscopy (TEM) to observe the cell morphology. PSC specimens for TEM were immersed in fixative (2.5\% glutaraldehyde) after washing three times with PBS. HSC specimens were washed with PBS and trypsin was added to the cultured cells. The cells were collected and immersed in fixative (2.5\% glutaraldehyde). Thereafter, the specimens were imaged by TEM (HT7700, HITACHI, JAPAN).

\section{Western blotting}

Extracellular proteins were extracted from cultured cell lysates with phosphatase and protease inhibitor cocktails. Protein $(20 \mu \mathrm{g})$ was separated from each sample by $6 \%$ or $10 \%$ sodium dodecyl sulfate polyacrylamide gel electrophoresis and transferred to a polyvinylidene difluoride membrane. The membranes were blocked using 5\% non-fatty milk in PBS containing $0.1 \%$ Tween- 20 for $1 \mathrm{~h}$ at RT, and they were incubated with primary antibodies overnight at $4^{\circ} \mathrm{C}$. Subsequently, the membranes were washed with PBS containing $0.1 \%$ Tween- 20 three times and then incubated with secondary antibodies conjugated with horseradish peroxidase for 90 min at RT. Protein bands were detected using a Bio-Rad Fluor-S Multilmager (Hercules, CA, USA). The band density was measured using Image software (version 
1.53, NIH, Bethesda, MD, USA). $\beta$-Actin served as a loading control. The antibodies used in the analysis were anti-Col-I (ab90395), anti-a-SMA (ab7817), and anti-OPN (ab8448; Abcam, Cambridge, UK).

\section{Detection of Col-囚, a-SMA, and OPN by ELISA}

Col-I, a-SMA, and OPN levels were measured using ELISA according to the manufacturer's instructions (CLOUD-CLONE CORP, CCC, USA). HSC-LX2 cells were treated with different ratios of PSCs for 24, 48, 72, and $96 \mathrm{~h}$. Next, the DMEM was collected and centrifuged at $1000 \times g$ for 20 min and the pellet was collected. The supernatant $(100 \mu \mathrm{L})$ was transferred to a new tube, and the levels of Col-I, a-SMA, and OPN were detected. The samples were then prepared and mixed with the standard sample in 96-well plates. The concentrations of Col-I, a-SMA, and OPN were determined by measuring the optical density at

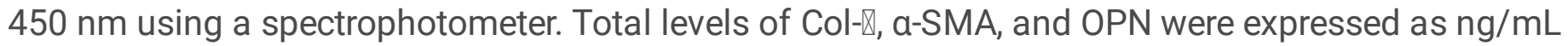
protein.

\section{Statistical analyses}

Data are presented as the mean \pm standard deviation. Data were assessed using GraphPad Prism 8.0 software (GraphPad, Inc., La Jolla, CA, USA) and one-way analysis of variance followed by Dunnett's various comparisons test.

\section{Results}

\section{Culture of HSC-LX2}

HSC-LX2 cells were cultured in DMEM for 3 days. After this period, and an increasing number of HSC-LX2 cells were observed in petri dishes. The cells also prominently developed fibroblastic morphology and contractile filaments during incubation, as shown in Fig. 1.

\section{HSC-LX2 promoted growth of AE-PSCs}

AE-PSCs were isolated and cultured with HSC-LX2 cells in DMEM for 3 days to evaluate the interaction between these cells. PSCs became highly motile, developed rapidly, and evaginated in the presence of HSC-LX2 [17]. In contrast, PSCs without HSC-LX2 grew slowly and were less developed, as shown in Figs. 2A and 2B. Thus, HSCs induced the growth of AE-PSCs. TEM was used to observe the structural changes in PSCs cultured with HSC-LX2 on days 3 and 4. The results showed that the cytoplasmic membrane of PSCs cocultured with HSC-LX2 had slightly changed, exhibiting increased thickness compared with the PSC control (Figs. 3D-3F). Slight changes in the cytoplasmic structure of the PSC control occurred because of cell growth in during culture (Figs. 3A, 3B, 3D, and 3E).

\section{Structural changes in HSC-LX2 treated with PSCs}

We used two different human HSC-LX2 cell lines. The first line was derived from normal human HSCLX2 as a control, and the second line was derived from PSC-treated human HSC-LX2 cells. HSCs were activated at ratios of 1:200 and 2:200 for 3 days, whereas HSCs were inhibited at a ratio of 3:200 
(Fig. 2A). Structural changes were analyzed by TEM on days 3 and 4, which revealed HSC-LX2 structural alterations. Lipid droplets were detected by TEM on day 0 and remained in normal human HSC-LX2 until days 3 and 4 (Figs. 4A-C). Lipid droplets were not observed in some cells, whereas they were degenerated in other cells on days 3 and 4 in PSC-treated human HSC-LX2 cells during the fibrosis transformation phase. This result reveals the fibrogenic transformation of these cells (Figs. 4D and 4E). Furthermore, the Golgi apparatus was larger than normal (Fig. 4D). Some changes in the cytoplasm were observed on day 3, with autophagosomes forming on day 4 in PSC-treated human HSC-LX2 cells that were later removed by exocytosis (Fig. 4E). The nuclei of HSCs also exhibited structural changes. HSCs had two or more nuclei on day 3 , and the nucleus began to fragmentation in HSCs on day 4 compared to in normal HSCs (Figs. 5A-E).

\section{AE-PSC-stimulated elevation of Col-】 in HSC-LX2}

Col- $\$ significantly affects liver fibrosis. Therefore, its expression in HSC-LX2 cells was examined in the presence of AE-PSCs. The expression of Col- $\triangle$ at different ratios of PSC: HSC-LX2 (1:200, 2:200, and 3:200) for 3 days was measured by western blotting (Fig. 6A).

These results were confirmed by ELISA. Col- $\$ levels were significantly higher in cells treated with AE-PSCs than in untreated HSCs. The 1:200 ratio of Col- $₫$ increased dramatically on days $2(P \otimes 0.05)$ and $3(P \square$ $0.01)$ (Fig. 6B). Additionally, for the 2:200 ratio, Col- $\$ was significantly increased on days $1(P<0.05), 2(P$

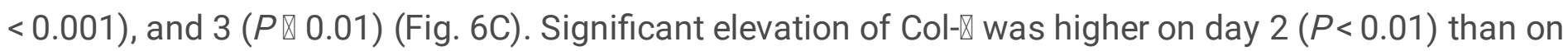
days $1(P<0.01)$ and $3(P<0.05)$ at a 3:200 ratio (Fig. 6D). Collectively, the Col- $\rrbracket$ expression level increased gradually on days 1 and 2 at ratios of 1:200 and 2:200 (Fig. 6E), whereas it continued to increase at a 1:200 ratio on day 3, but began to decrease at 2:200 and 3:200 ratios because of the large numbers of PSCs that consumed nutrients and effect of IPSC secretion on HSC-LX2 growth (Fig. 6F).

\section{HSCs presented increased a-SMA expression after treatment with AE-PSCs}

AE-PSCs have been reported to play an essential role in the activation and proliferation of HSCs in coculture accompanied by a-SMA expression. a-SMA expression in treated HSCs was measured by western blotting (Fig. 7A) and confirmed by ELISA (Fig. 7B). Figure 7B shows that the levels of a-SMA expression increased significantly at a ratio of $1: 200$ on days $2(P<0.01)$ and days $3(P<0.01)$. A significant increase in a-SMA expression was observed for the 2:200 ratio on days 1,2 , and $3(P<0.05, P$ $<0.01$, and $P<0.05$, respectively) (Fig. 7C). Additionally, significant elevation of a-SMA was observed for 3:200 on days 1,2 , and $3(P<0.01, P<0.001$, and $P<0.05$, respectively) (Fig. 7D). a-SMA expression on day 1 was significant at the 2:200 and 3:200 ratios but not significant at the 1:200 ratio (Fig. 7E). The expression of a-SMA on day 2 was significant at a 2:200 ratio, followed by 1:200 and then 3:200 (Fig. 7F). a-SMA expression on day 3 was moderate at a ratio of 1:200, and the level of protein expression at the 2:200 and 3:200 ratios was decreased (Fig. 7G).

Effect of AE-PSCs on expression of OPN 
In addition to Col- $\$ and a-SMA, OPN is a major ECM protein produced during fibrosis. Human HSCs with PSCs were incubated in coculture to identify the role of PSCs in OPN expression. Western blotting and ELISA were performed to measure OPN expression: OPN expression in treated HSCs was measured by western blotting (Fig. 8A).

Moreover, ELISA showed that OPN expression was increased in the coculture of HSC-LX2 with PSCs during incubation for 4 days. PSCs were added to stimulate OPN synthesis (Figs. 8A-C). A significant relationship was observed between treated and non-treated HSCs. This relationship at 1:200 was significant on days $2(P<0.01), 3$, and $4(P<0.001)$ (Fig. 8B); for 2:200, the results were significant on days $1(P<0.05), 3(p<0.001)$, and 2 and $4(P<0.01)$ (Fig. 8C). Moreover, at 3:200, this relationship was significant on days $1(P<0.01), 2(P<0.001)$, and 3 and $4(P<0.001)$ (Fig. 8D). However, the OPN level at 1:200 gradually increased and started decreasing on days 3 and 4 for 2:200 and 3:200, respectively. The decrease was significant at a 3:200 ratio, as shown in Fig. 8D.

\section{Discussion}

We evaluated the potential interactions of HSC-LX2 and AE-PSCs. Our results revealed that AE-PSCs activate HSC-LX2 and that HSC-LX2 effects secretion by PSCs in coculture. The development of cocultured PSCs with HSC-LX2 was better than that of control cells. PSCs were evaginated and showed rapid motility in coculture, whereas PSC control cultures grew slowly and invaginated on day 1 . Moreover, the cytoplasmic content changed in the PSC control after continuous development for 4 days. Cytoplasmic membrane changes were observed in cocultured PSCs. This result may have occurred because of the secretion of HSC-LX2, which affected the cytoplasmic membrane. Li et al. (2018) explained the development of PSCs in hepatocyte culture systems and observed that PSCs showed rapid motility and were evaginated in the first days of culture. HSCs treated with PSCs did not contain lipid droplets compared to HSC controls. The number of lipid-containing HSCs decreased significantly during the transformation phase of liver cirrhosis, indicating that the cells underwent fibrogenic transformation [18]. All features resulting from treatment of HSCs with PSCs, including multiplication or disappearance of the nucleus, enlargement of the Golgi apparatus, changes in the cytoplasm, and finally, transformation of cytoplasm into autophagosomes, indicate fibrogenic transformation compared to the HSC controls.

HSCs contain one or more oval-shaped nucleoli. These morphological changes of the nucleoli were observed in HSC culture, which display thin, multiple, and elongated processes that extend from the cell body [19]. Activation of human HSCs results in expression of a-SMA, which is a specific marker of activated HSCs [20].

Our results are similar to those of Budke et al., in which PSC reproduction was stimulated by bile salts in the intestine. Hepatocytes may produce bile, thereby stimulating PSC evasion after their release from cysts [21]. Our study revealed that HSCs produce Col- $\bigotimes$, which promotes the development of AE-PSCs. Previous studies also confirmed that collagen-integrated liver cells form bile salts in response to PSCs [21-22]. In this study, PSCs induced HSCs after $72 \mathrm{~h}$, particularly at high ratios, compared with control 
cells that grew favorably and showed increased survival. This result is similar to that obtained in a previous study of the effect of Echinococcus species PCSs on the process of fibrosis [23]. Additionally, PSCs inhibit the spread of HSCs by directly targeting TGF-BR囚/囚 [24]. Cystic echinococcosis cyst fluid can inhibit the proliferation of HSCs and increase the main markers of HSCs, including Col- $\triangle$ and a-SMA [23].

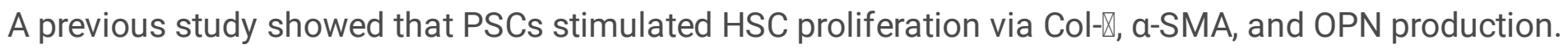
HSCs are typically static but become fibroblasts and accumulate in the ECM when activated during liver injury [8]. Thus, activation of HSCs is an important process in cirrhosis. Col- $\triangle$ and a-SMA increased at different ratios for different incubation periods. Previous studies also showed similar results for P1CP and $3 \mathrm{H}$-Pro. Collagen synthesis and a-SMA levels increased after different incubation periods $[8,15]$. Our experiments confirmed that OPN expression increased in HSCs treated with AE-PSCs. These results agree with those of another report on Hh signaling in liver cirrhosis. Overall, OPN and Hh are key markers of liver cirrhosis [26].

Many studies have demonstrated the role of HSCs in the synthesis of ECM components, fibrosis, and cirrhosis in bovine livers infected with helminths, such as Fasciola hepatica and Dicrocoelium dendriticum. HSCs play an important role in the development of fibrosis and other stages of cirrhosis [23, 27]. TGF- $\beta$ is the main factor that activates HSC and collagen deposition, as it is necessary for the differentiation of HSCs. Cirrhosis is a common progressive pathological process that occurs after extensive liver injury. ECM deposits are characteristic features of liver cirrhosis observed after hepatic cell activation. HSCs differentiate and become the main producers of the $\operatorname{ECM}[25,27]$.

\section{Conclusion}

We explored the interaction between treated HSCs and AE-PSCs, which revealed that AE-PSCs induced liver fibrosis through HSC-producing fibrosis proteins (i.e., Col-囚, a-SMA, and OPN). These findings may contribute to understanding the pathogenesis of parasitic hepatic fibrosis. Additional studies are needed to focus on the parasite and its participation in this complicated process to confirm and explain the interaction between AE-PSCs and the host.

\section{Declarations}

\section{Acknowledgements}

We would like to thank Editage (www.editage.cn) for English language editing.

\section{Funding}

This work was supported by Guangxi Zhuang Autonomous Region Science and Technology Department Project (grant 2020JJA140073) and Qinghai Science and Technology Department Project (grant 2019-SF131). 


\section{Authors' Contributions}

CDP oversaw the laboratory work, conceived and designed the study, and drafted the manuscript. ES performed the laboratory work. JBF and ZYG assisted with the laboratory work. MASD provided writing assistance.

\section{Ethics approval and consent to participate}

This study was approved by the ethics review board of the Affiliated Hospital of Qinghai University (approval number: P-SL-2019054).

\section{Consent for publication}

Not applicable.

\section{Availability of data and materials}

The datasets used in this study are available from the corresponding author on reasonable request.

\section{Competing interests}

The authors declare that they have no competing interests.

\section{References}

1. Gottstein B, Wang JH, Blagosklonov O, Grenouillet F, Millon L, Vuitton DA, et al. Echinococcus metacestode: in search of viability markers. Parasite. 2014;21:

2. Brehm K, Koziol U. Echinococcus-host interactions at cellular and molecular levels. Advances in parasitology. Adv Parasitol. 2017;95:147-212.

3. Craig PS, McManus DP, Lightowlers MW, Chabalgoity JA, Garcia HH, Gavidia CM, et al. Prevention and control of cystic echinococcosis. Lancet Infect Dis. 2007;7(6):385-94.

4. Brunetti E, Kern P, Vuitton DA. Expert consensus for the diagnosis and treatment of cystic and alveolar echinococcosis in humans. Acta Trop. 2010;114(1):1-16.

5. Hemphill A, Stadelmann B, Scholl S, Müller J, Spiliotis M, Müller N, et al. Echinococcus metacestodes as laboratory models for the screening of drugs against cestodes and trematodes. Parasitology. 2010;137(3):569-87.

6. Zhang W, Zhang Z, Wu W, Shi B, Li J, Zhou X, et al. Epidemiology and control of echinococcosis in central Asia, with particular reference to the People's Republic of China. Acta Trop. 2015, 141:235-43. 
7. Brunetti E, Garcia HH, Junghanss T. Cystic echinococcosis: chronic, complex, and still neglected. PLoS Negl Trop Dis. 2011;5(7):e1146.

8. Gottstein B, Hemphill A. Immunopathology of echinococcosis. Chem Immunol. 1997;66:177-208.

9. McManus DP, Zhang W, Li J, Bartley PB. Echinococcosis. Lancet. 2003;362(9392):1295-304.

10. Moro P, Schantz PM. Echinococcosis: a review. International journal of Infectious diseases. 2009;13(2):125-33.

11. Lu Y, Huang Q, Zhou Z, Lu Y. An overview of parasitic diseases by analyzing publications at National Institute of Parasitic Diseases Control and Prevention in the last five years. Chin J Dis Control Prev. 2015;19:86-90.

12. Beschin A, De Baetselier $P$, Van Ginderachter JA. Contribution of myeloid cell subsets to liver fibrosis in parasite infection. J Pathol. 2013;229(2):186-97.

13. Förster S, Koziol U, Schäfer T, Duvoisin R, Cailliau K, Vanderstraete M, et al. The role of fibroblast growth factor signalling in Echinococcus multilocularis development and host-parasite interaction. PLoS Negl Trop Dis. 2019;13(3):e0006959.

14. Mohammed A, Allen J, Rogan M. Echinococcus granulosus cyst fluid enhances epithelialmesenchymal transition. Parasite Immunol. 2018;40(6):e12533.

15. Wang J, Zhang C, Wei X, Blagosklonov O, Lv G, Lu X, et al. TGF- $\beta$ and TGF- $\beta /$ Smad Signaling in the Interactions between Echinococcus multilocularisand Its Hosts. PLoS ONE 2013;8(2):e55379.

16. Moazeni M, Saharkhiz MJ, Hosseini AA. In vitro lethal effect of ajowan (Trachyspermum ammi L.) essential oil on hydatid cyst protoscoleces. Vet Parasitol. 2012;187(1-2):203-8.

17. Li L, Chen B, Yan H, Zhao Y, Lou Z, Li J, et al. Three-dimensional hepatocyte culture system for the study of Echinococcus multilocularis larval development. PLoS Negl Trop Dis. 2018;12(3):e0006309.

18. Nepomnyashchikh GI, Aidagulova SV, Nepomnyashchikh DL, Kapustina VI, Postnikova OA. Ultrastructural and immunohistochemical study of hepatic stellate cells over the course of infectious viral fibrosis and cirrhosis of the liver. B Exp Biol Med. 2006;142(6):723-8.

19. Herrmann J, Axel M. Gressner A M, Weiskichen R. Immortal hepatic stellate cells lines: useful tools to study hepatic stellate cell biology and function? J Cell Mol Med. 2007;11(4):704-22.

20. Chang $\mathrm{K}-\mathrm{T}$, Tsai $\mathrm{M}-\mathrm{J}$, Cheng $\mathrm{Y}-\mathrm{T}$, Chen $\mathrm{J}-\mathrm{J}$, Hsia R-H, Lo $\mathrm{Y}-\mathrm{S}$, et al. Comparative atomic force and scanning electron microscopy: an investigation of structural differentiation of hepatic stellate cells. $J$ Struct Biol. 2009;167(3):200-8.

21. Budke CM, Deplazes P, Torgerson PR. Global socioeconomic impact of cystic echinococcosis. Emerg Infect Dis. 2006;12(2):296.

22. Lee UE, Friedman SL. Mechanisms of hepatic fibrogenesis. Best Pract Res Clin Gastroenterol. 2011;25(2):195-206.

23. Niu F, Chong S, Qin M, Li S, Wei R, Zhao Y. Mechanism of fibrosis induced by Echinococcus spp. Diseases. 2019;7(3):51. 
24. Kwiecinski M, Noetel A, Elfimova N, Trebicka J, Schievenbusch S, Strack I, et al. Hepatocyte growth factor (HGF) inhibits collagen I and IV synthesis in hepatic stellate cells by miRNA-29 induction. PloS ONE. 2011;6(9):e24568.

25. Bartel DP. MicroRNAs: target recognition and regulatory functions. Cell. 2009;136(2):215-33.

26. Petrova R, Joyner AL. Roles for Hedgehog signaling in adult organ homeostasis and repair. Development. 2014;141(18):3445-57.

27. Boldin MP, Baltimore D. MicroRNAs, new effectors and regulators of NF-KB. Immunol Rev. 2012;246(1):205-20.

\section{Figures}

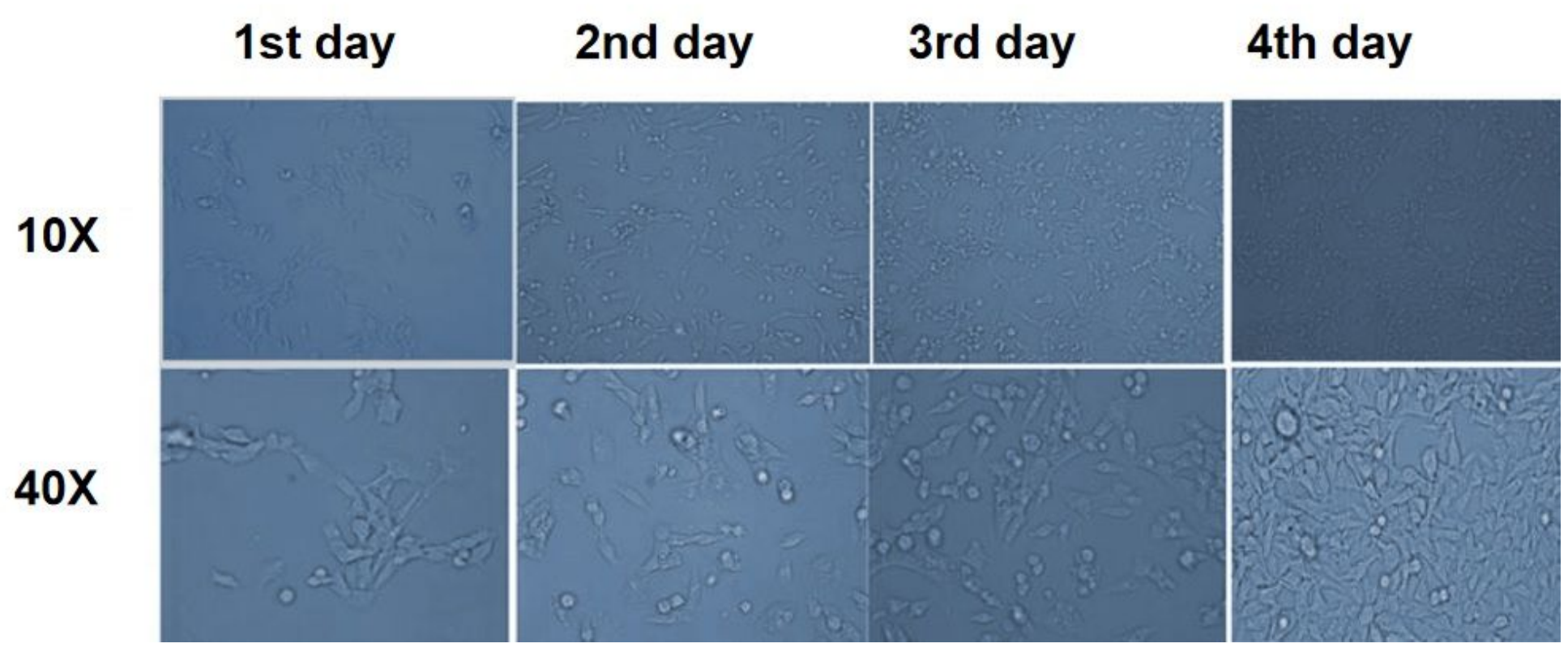

Figure 1

Morphological images of activated HSC-LX2. These images were taken after 24, 48 and $72 \mathrm{~h}$. 
A
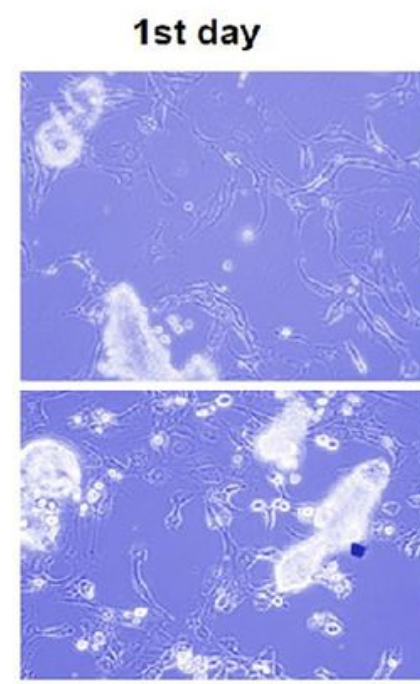

Ratio

2:200

Ratio

3:200
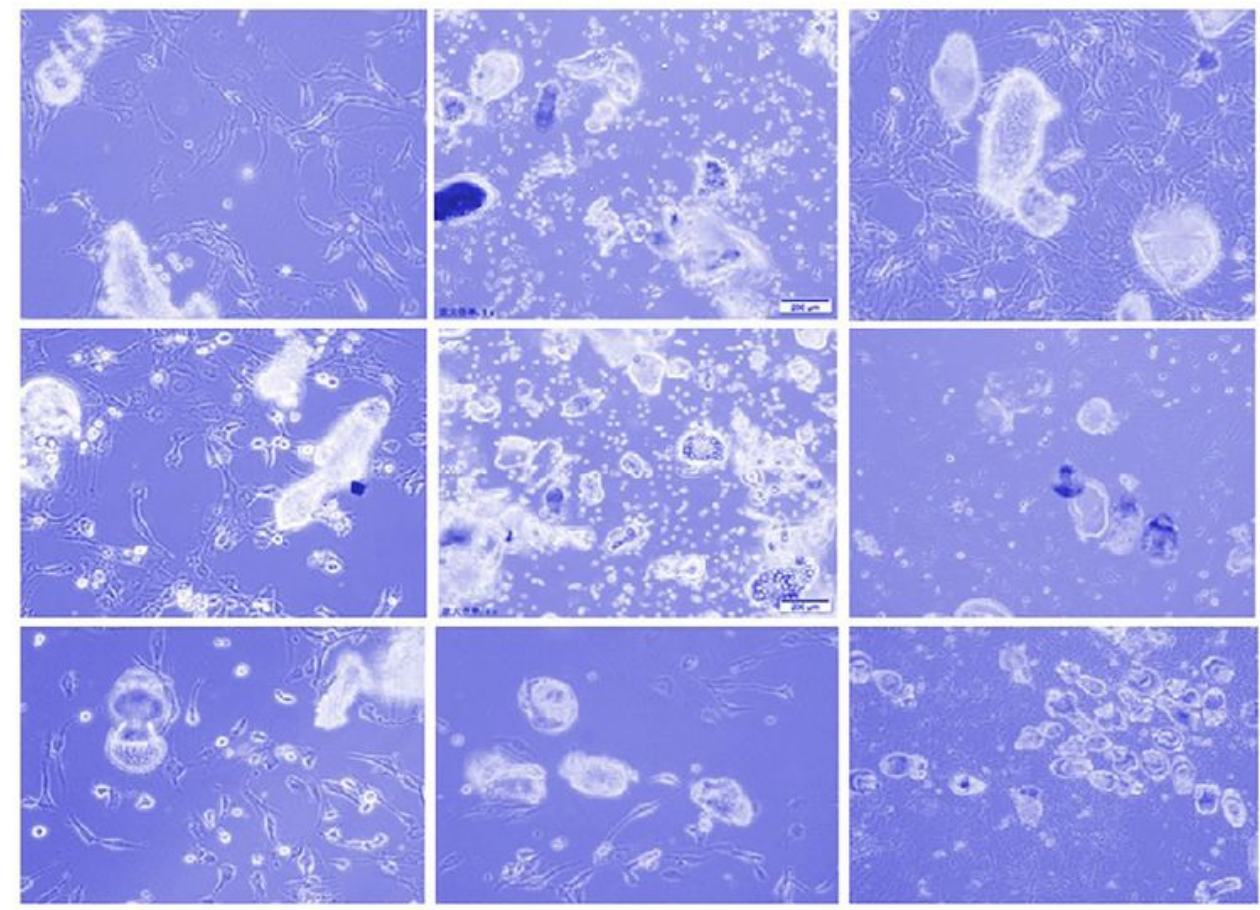

B

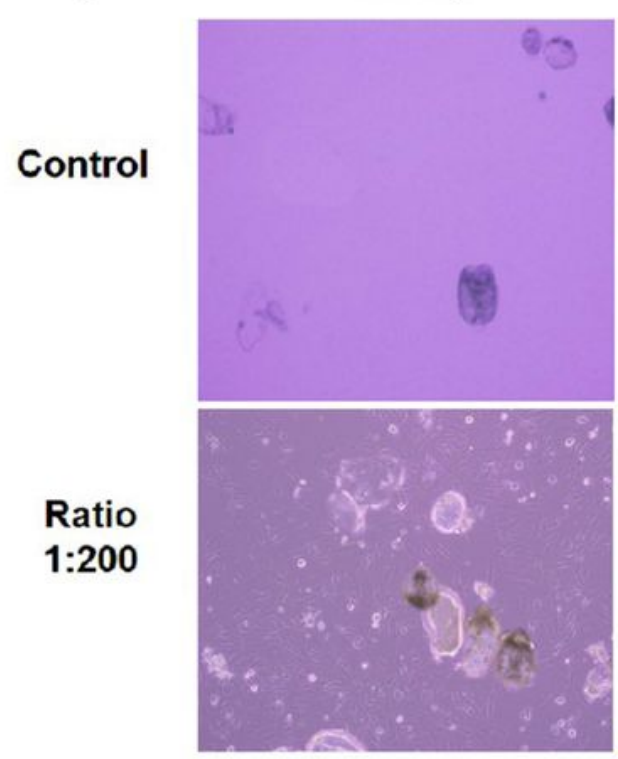

$10 \mathrm{X}$

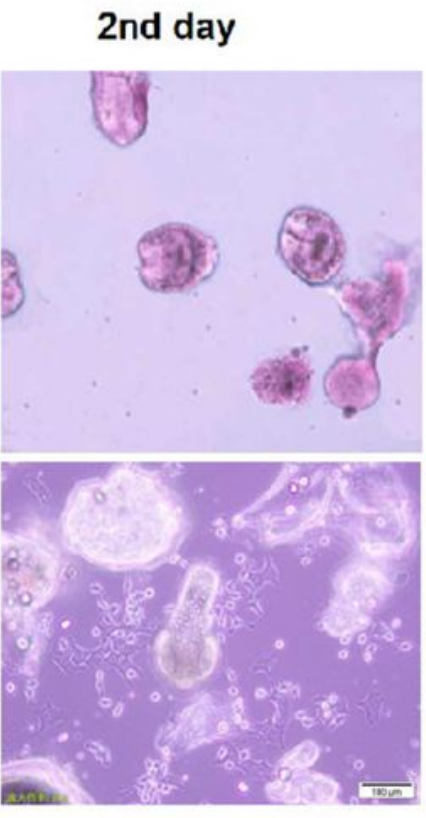

$10 X$

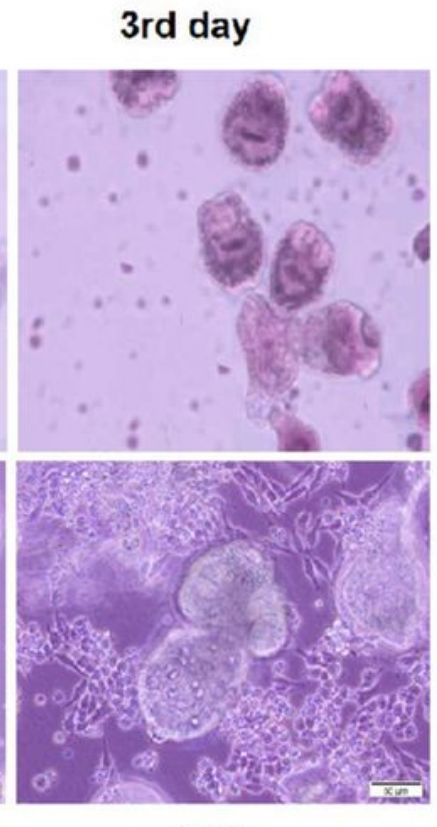

$20 X$

\section{Figure 2}

(A) Morphological images of HSCs with PSCs in culture media. These images were taken after 24,48, and $72 \mathrm{~h}$. Different ratios of PSCs 1-(1:200): 1 PSCs and $200 \mathrm{HSCs}, 2-(2: 200): 2$ PSCs and $200 \mathrm{HSCs}$, and 3(3:200): 3 PSCs and 200 HSCs compare to Control in figure 2B. (B) Light microscopy images show the development of Echinococcus multilocularis (EM) PSCs and human HSCs cultured in vitro. Ratio (1:200) group evaginated PSCs compared with the control group. 

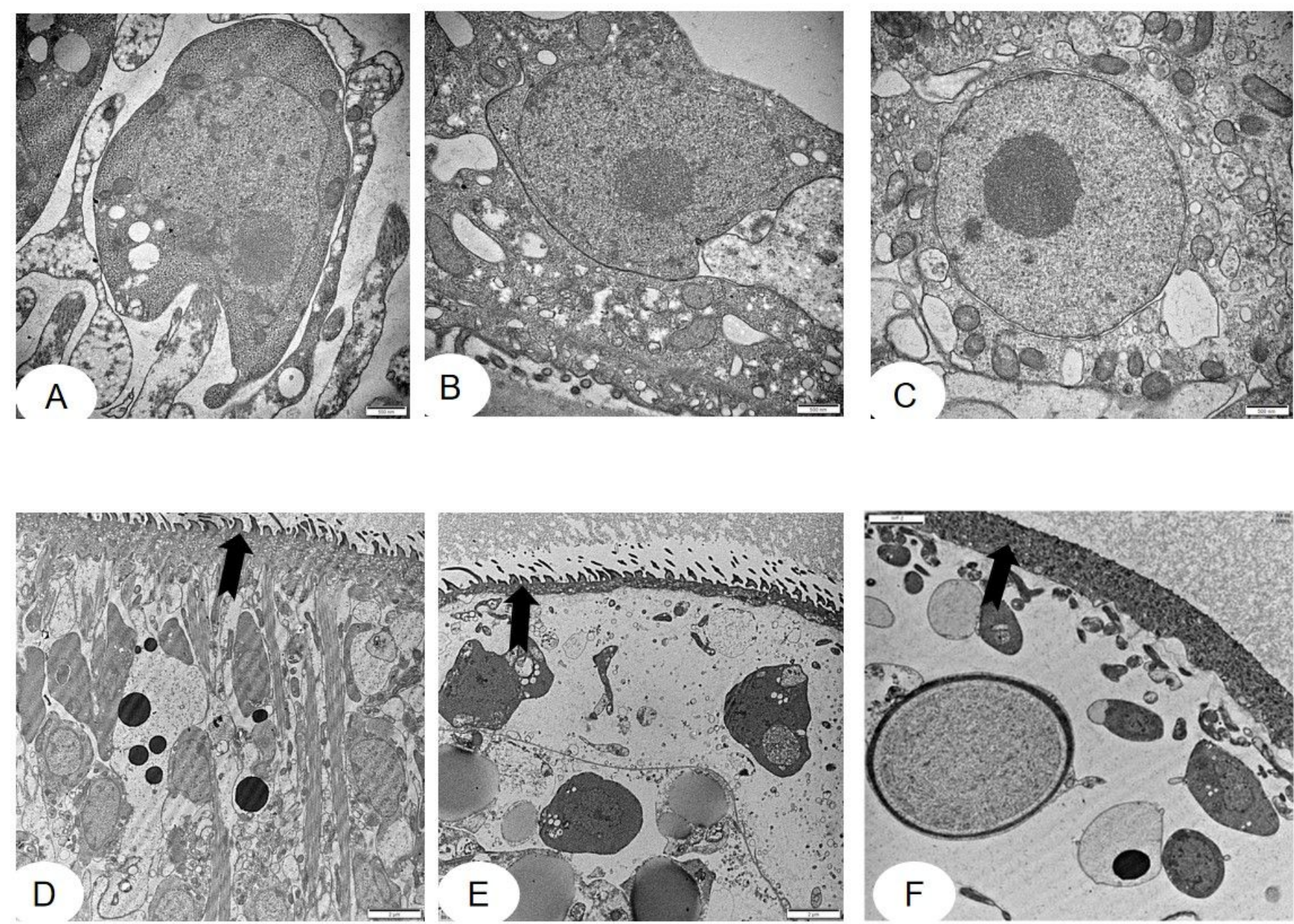

\section{Figure 3}

The internal structure of PSC (TEM, 30 000x). (A) Cytoplasmic structure on the 0 day in HSCs control. (B) Cytoplasm structure on the 4th day in HSCs control. (C) Cytoplasm structure on the 4th day in human HSCs treated with PSCs. (D) The cytoplasmic membrane was prominent on the 0 day in HSCs control. (E) The cytoplasmic membrane was prominent on the 4th day in HSC control. (F) The prominent cytoplasmic membrane disappeared on the 4th day in THSCs. 

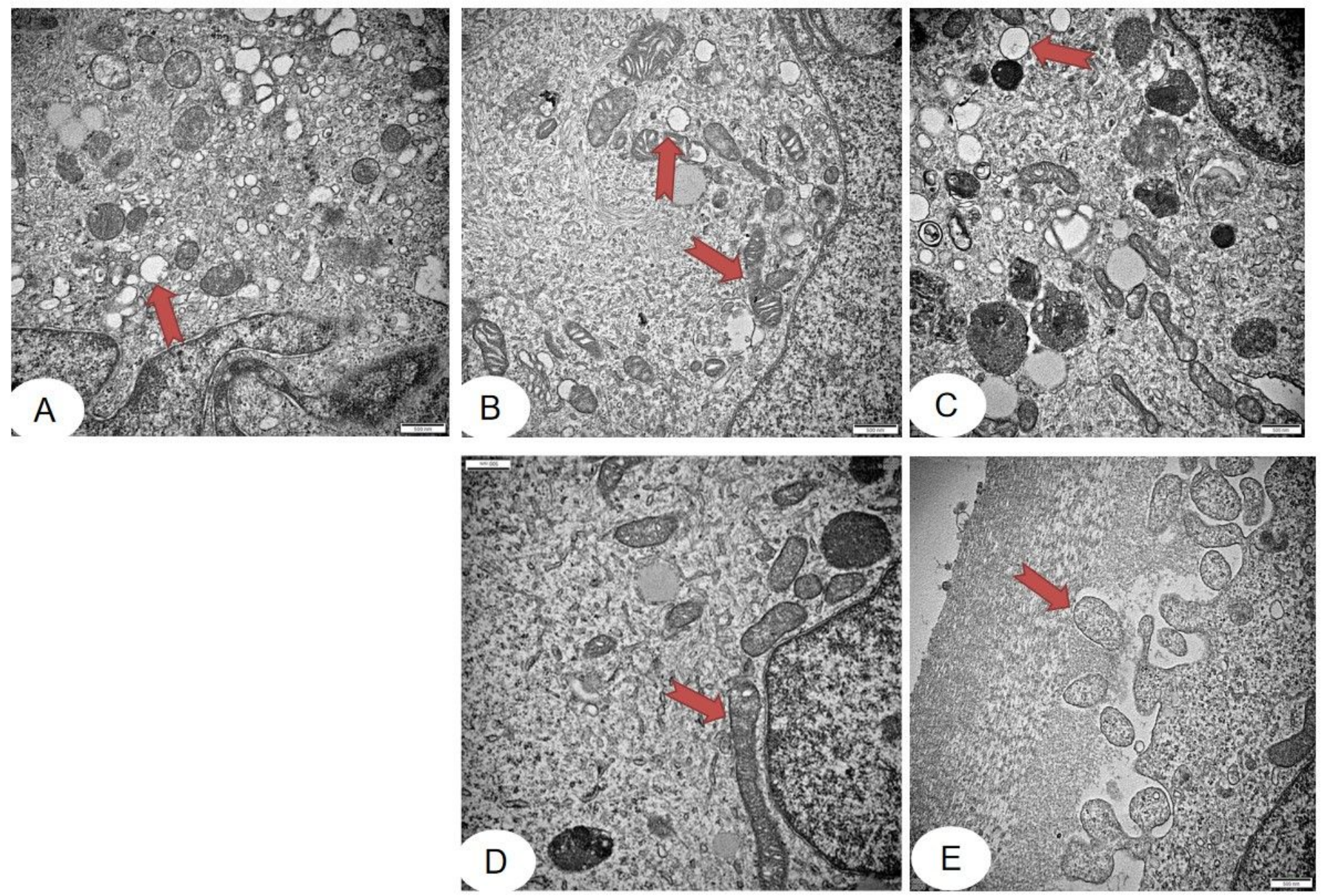

\section{Figure 4}

Structural characteristics of HSCs (TEM, 30 000x). (A, B, and C) The lipid droplets' structures of oval shape appear in control HSCs (arrowheads). (D) The lipid droplets structures of oval shape disappeared in THSCs (HSCs treated with PSCs), Golgi apparatus was larger than in control HSCs, and some changes in cytoplasm are observed compared with the control on the 3rd day (arrowheads). (E) The lipid droplets structures of oval shape disappeared in THSCs, and the cytoplasm appeared as autophagosomes in THSCs on the 3rd day (arrowheads). 

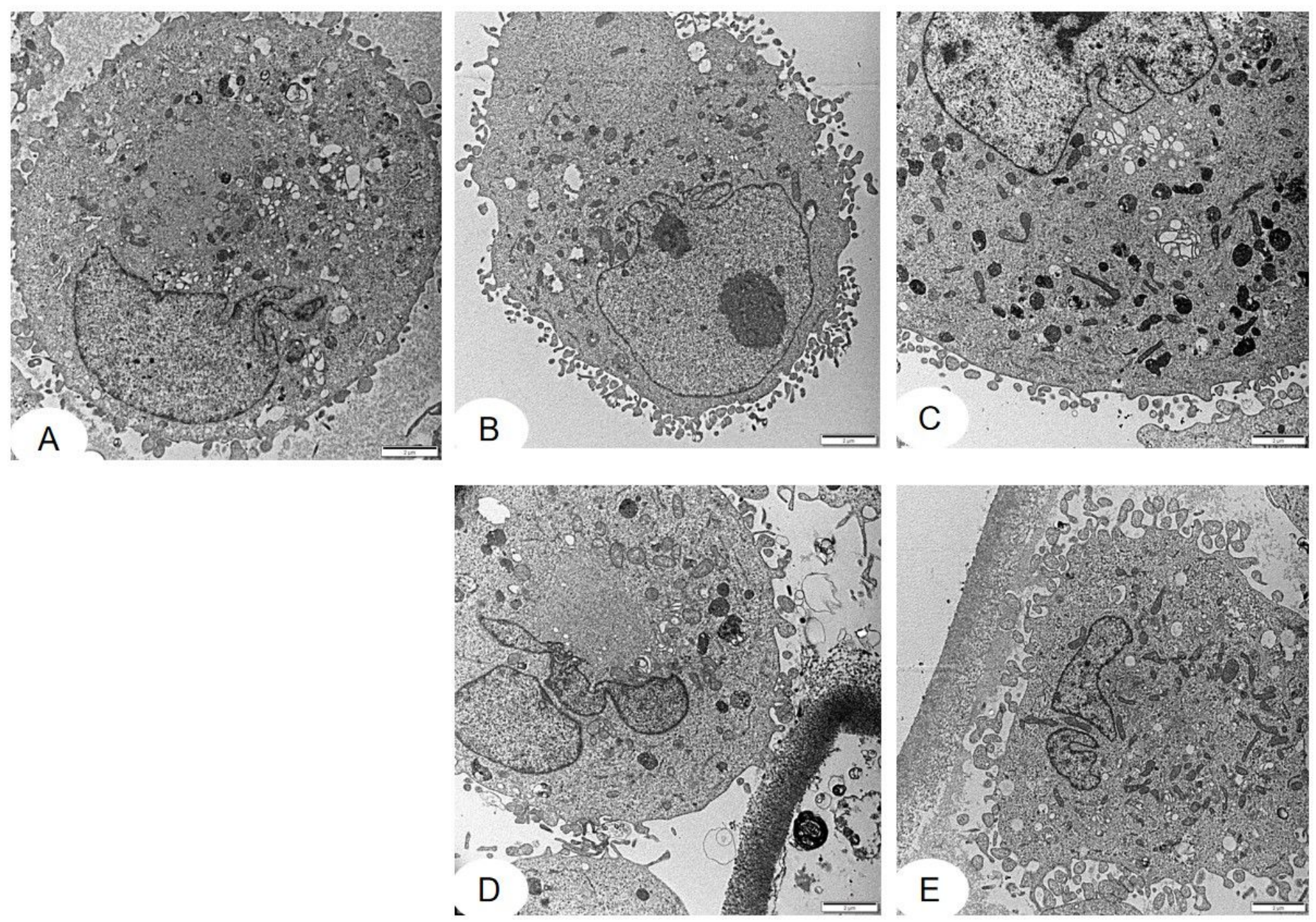

\section{Figure 5}

Structural characteristics of HSCs (TEM, $10000 \times$ ). (A, B, and C) on the 1st, 3rd, and 4th days, respectively as control. The nucleus structure in control HSCs: (D) The nuclei divided into two nuclei on the 3rd day. (E) The nucleus started to vanish on the 4th day. 


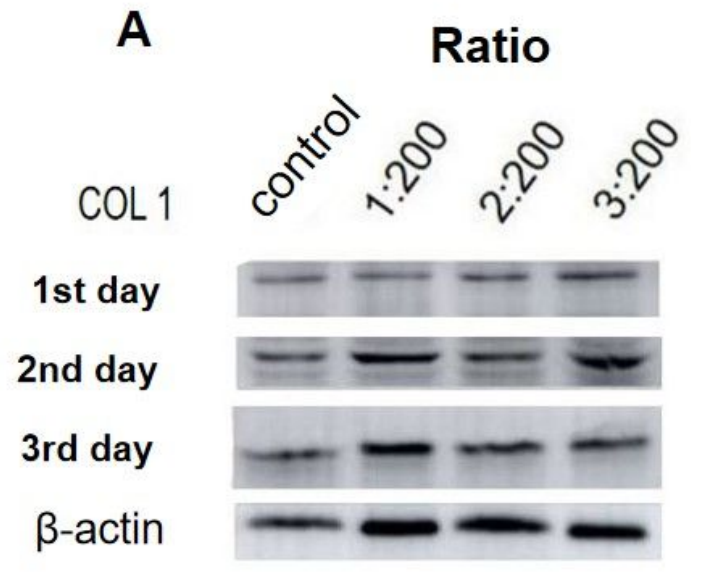

B

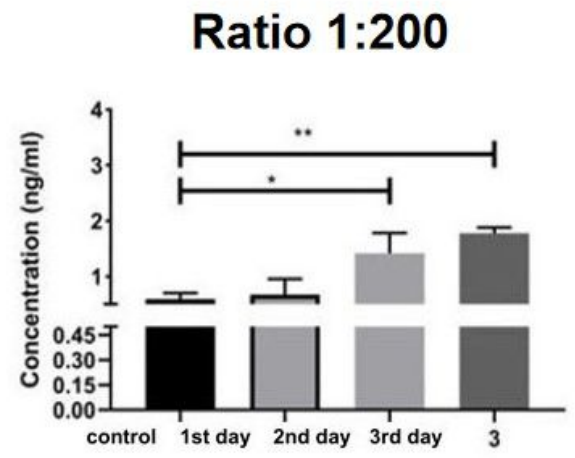

Ratio 2:200

D

E

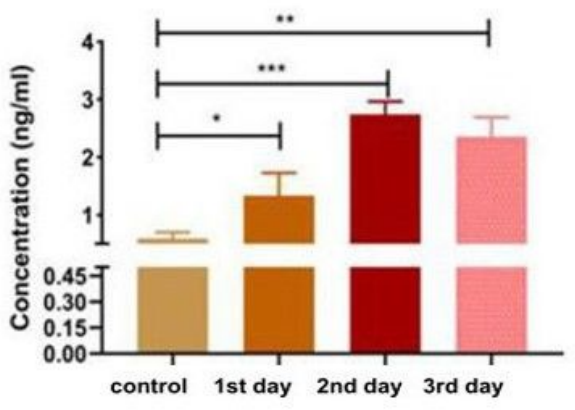

$\mathbf{F}$

\section{Ratio 3:200}
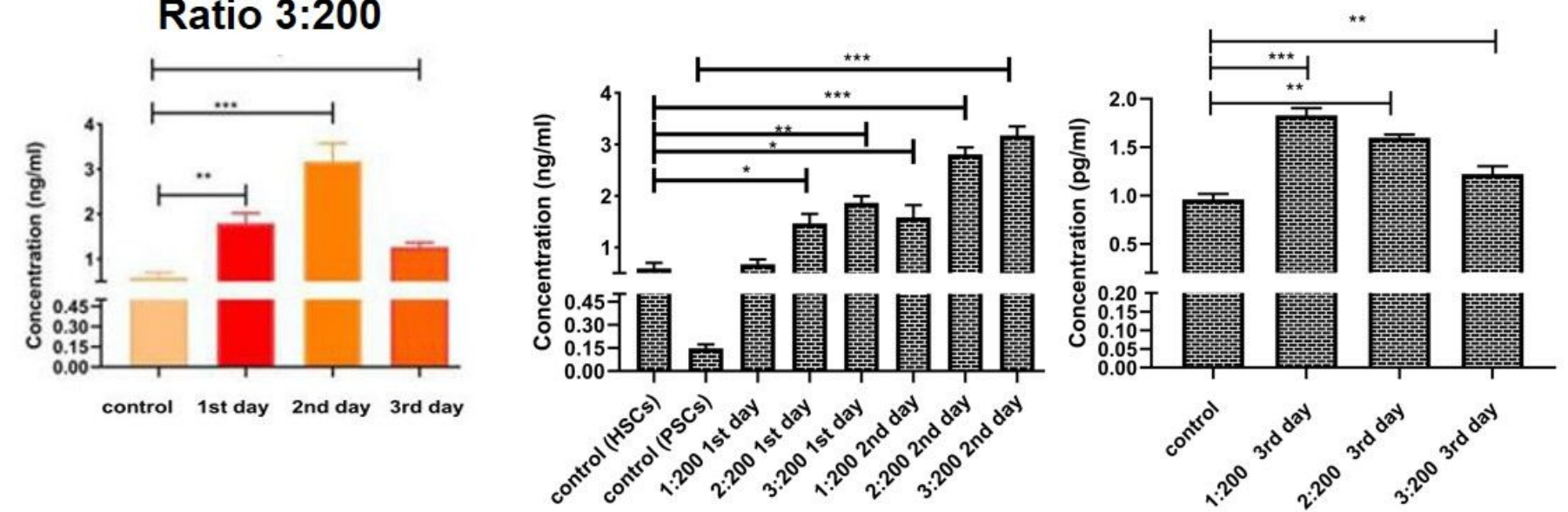

Figure 6

Col- $\bigotimes$ expression profile measured by Western blot (WB) and Elisa (A) expression of Col- $\$ assessed by WB.

(B) ratio (1:200), Col- $\$ expression was significant on days 2 and 3 but was not significant on day 1. (C) Col- $₫$ expression at ratio (2:200) was significant on days 1, 2, and 3. (D) Deceased Col- $₫$ expression on day 3 at a ratio of 3:200 and continued to increase on days 1 and 2. (E) Col- $\$ marker expression in

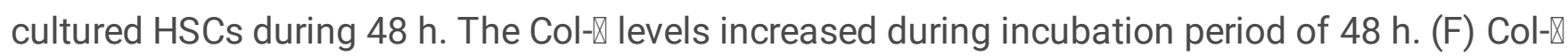
expression on day 3 was increasing at 1:200 and started to decrease at 2:200 and 3:200 ratios. 
a-SMA

1st day

2nd day

3rd day

$\beta$-actin

B

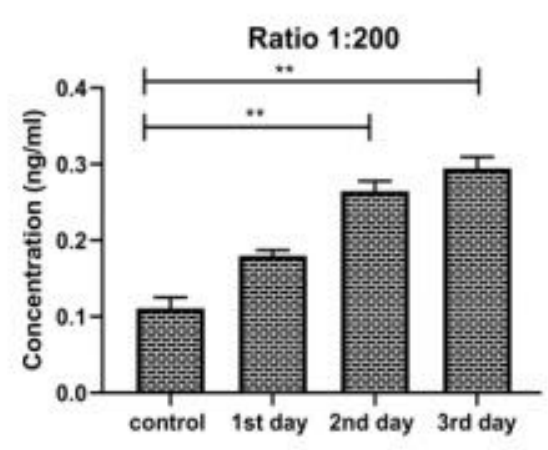

E

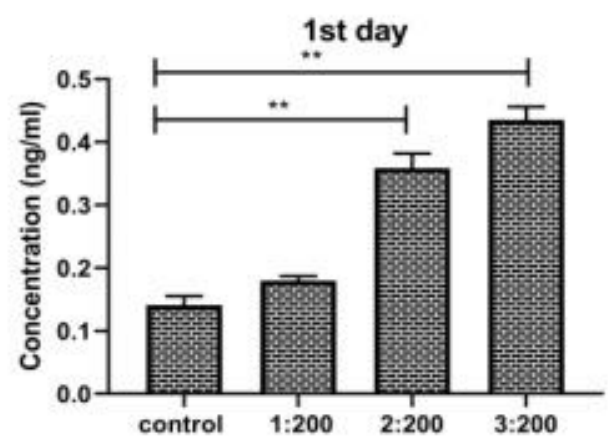

Ratio
C

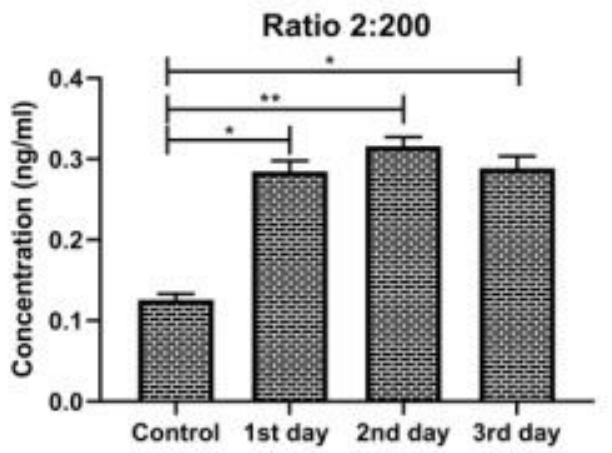

F

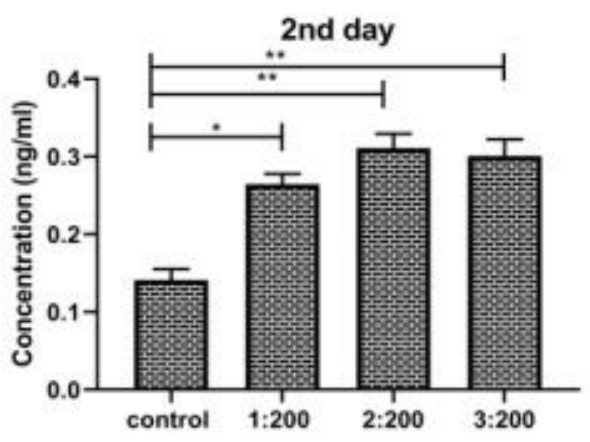

Ratio
D

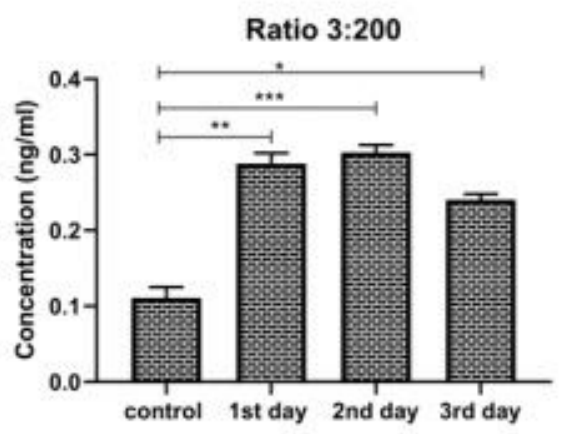

G

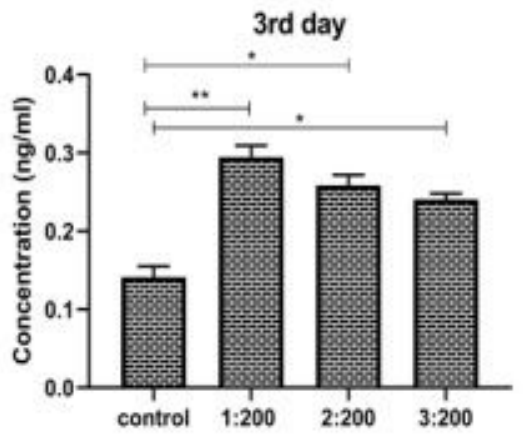

Ratio

Figure 7

a-SMA expression profile measured by WB and ELISA (A) a-SMA expression assessed by WB. (B) a-SMA expression was significant on the 2nd and 3rd days and was not significant in the 1st day for the ratio of 1:200. (C) a-SMA expression was significantly on the 1 st, 2 nd, and 3rd days for the ratio 2:200. (D) Deceased a-SMA expression was observed on the 3rd day for the ratio of 3:200, and continued increase of a-SMA expression on the 1st and 2nd day. (E) Expression of a-SMA marker in cultured HSCs during 24 h. The a-SMA levels was increasing during incubation period of $24 \mathrm{~h}$. (F) a-SMA expression on the $2 \mathrm{nd}$ 
day was increasing at all ratios, i.e., 1:200, 2:200, and 3:200. (G) a-SMA expression on the 3rd day continued to increase at the ratio of 1:200 and started to decrease at 2:200 and 3:200.

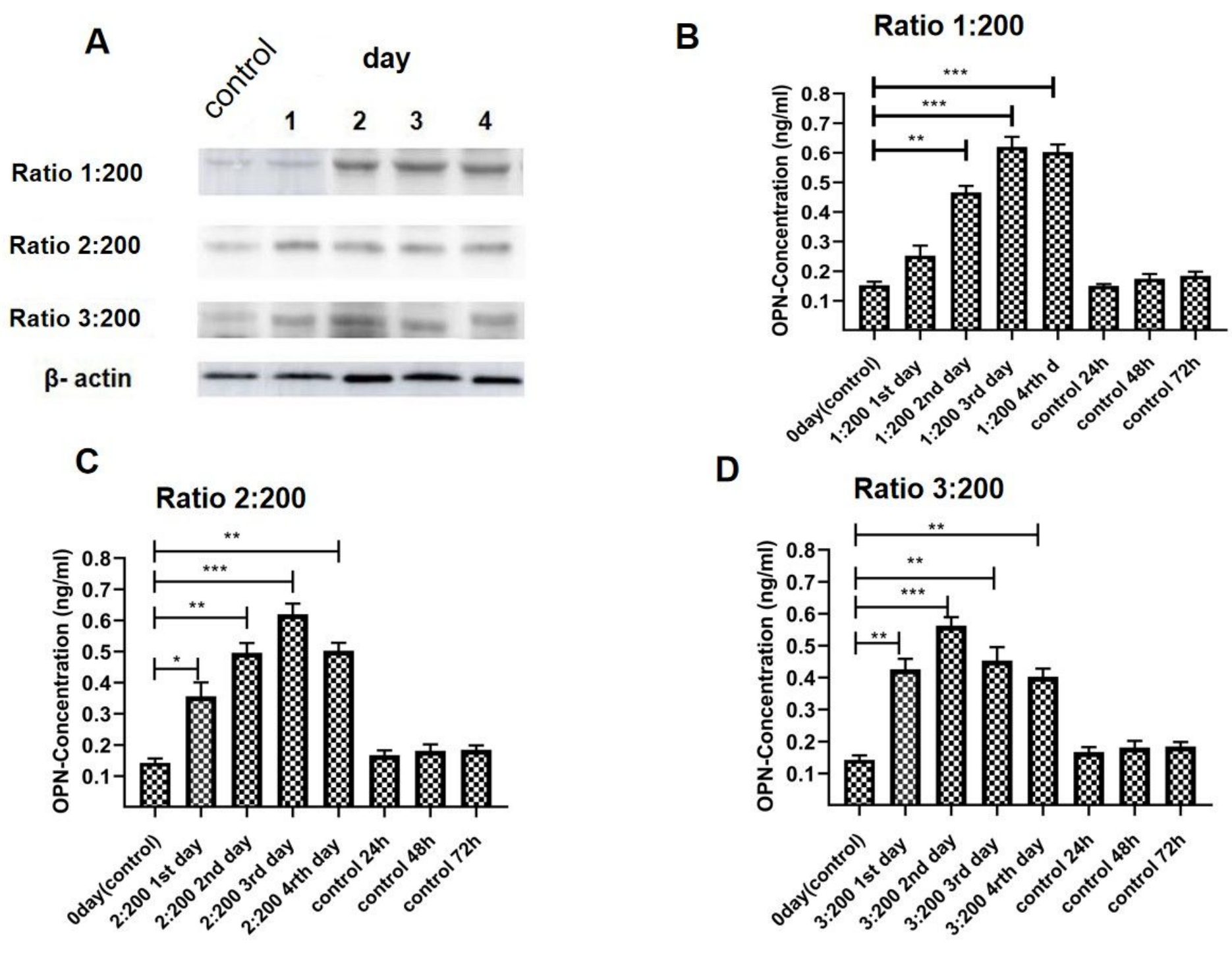

Figure 8

Osteopontin (OPN) expression profile measured by WB and Elisa. (A) Expression of OPN assessed by WB. (B) OPN expression was significant on the $2 \mathrm{nd}$, $3 \mathrm{rd}$ and 4 th day and was not significant on the 1 st day for ratio (1:200). (C) Ratio (2:200) showed significant OPN expression on the $1 \mathrm{st}$, 2 nd and 3rd days. (D) Decrease of OPN expression on the 3rd and 4th days at a ratio of 3:200, whereas the continued increase of OPN expression was observed on the 1 st and 2 nd day.

\section{Supplementary Files}

This is a list of supplementary files associated with this preprint. Click to download.

- Graphicalabstract.jpg 